\title{
A NOVEL METHOD FOR SIMULATING THE COMPLEX GINZBURG-LANDAU EQUATION
}

\author{
BY \\ DANIEL GOLDMAN AND LAWRENCE SIROVICH \\ Brown University, Providence, Rhode Island
}

\begin{abstract}
We present a split-step method for integration of the complex GinzburgLandau equation in any number of spatial dimensions. The novel aspect of the method lies in the fact that each portion of the splitting is explicitly integrable. This leads to an extremely fast, stable, and efficient procedure. A comparison is made with spectrai anci pseudospectral procedures which have appeared in the literature.
\end{abstract}

1. Introduction. The problem of interest is the $d$-dimensional complex GinzburgLandau equation

$$
\frac{\partial A}{\partial t}=q^{2}\left(c_{0}+i\right) \nabla^{2} A+\rho A-(\rho-i)|A|^{2} A
$$

subject to periodic boundary conditions on $[0,2 \pi]^{d}$. Here $A(\mathbf{x}, t)$ is a complex amplitude; $\mathbf{x}$ and $\nabla^{2}$ are the $d$-dimensional spatial variable and the Laplacian, respectively; and the parameters $q, \rho$, and $c_{0}$ are all assumed to be real and positive. For certain parameter sets, solutions of Eq. (1.1) exhibit spatiotemporal chaos or "turbulence".

We are interested in numerically studying turbulent solutions of Eq. (1.1) in one, two, and higher dimensions. In particular, we wish to examine the behavior in the limit $\rho=c_{\mathrm{C}} \downarrow 0$ for fixed $q \ll 1$. This regime, known as the dissipationless or nonlinear Schroedinger limit, has been studied analytically by Bartuccelli et al. [1, 2, 3], who have suggested that hard turbulence occurs here in two dimensions but not in one dimension. Since we desire high spatial accuracy, the calculation requires a large number of modes. Sirovich, Rodriguez, and Knight [15] used a pseudospectral method to study turbulence in the one-dimensionai CGL equation, while the twodimensional case was investigated numerically by Gaponov-Grekhov and Rabinovich [7] using a finite-difference scheme and by Coullet, Gil, and Lega [5] using a spectral method.

We have found a novel method for numerically integrating Eq. (1.1) which is more efficient and simpler to implement than the standard schemes, while giving the same spatial and temporal accuracy as spectral or pseudospectral schemes. Our method

Received November 23, 1992.

1991 Mathematics Subject Classification. Primary 65N99, 76E30, 35Q55, 58F05.

Key words and phrases. Split-step, Ginzburg-Landau, operator splitting methods.

(C) 1995 Brown University 
involves splitting Eq. (1.1) into two equations, each of which can be solved exactly, and then using the two analytical solutions to construct an approximate solution of Eq. (1.1). We derive schemes of this type which are first-order and second-order accurate in time and discuss their implementation. Verification is presented that our second-order method has excellent numerical stability and computational efficiency.

Our method is essentially the split-step Fourier algorithm, first presented by Tappert [17] in the context of solving a class of generalized Korteweg-de Vries equations and then extended by Hardin and Tappert [8] to a larger class of wave equations. In these cases, however, the nonlinear part of the problem was not explicitly solvable and hence required the use of a finite-difference scheme. Variations of this method have been used by other authors to study nonlinear wave problems numerically $[9$, $11,13,14,18]$. Bernoff [4] apparently used a split-step method to study the CGL equation but did not give a derivation.

We note that our numerical method is related to the symplectic integrators (see $[6,12,20])$ used for Hamiltonian systems. In particular, our second-order splitting scheme for the CGL equation corresponds to the symplectic scheme for the nonlinear Schroedinger equation [10] which was studied by Weideman and Herbst [19] and shown by Taha and Ablowitz [16] to be superior to other methods for the NLS equation.

2. Splitting and analytical solutions. Our numerical method is based on splitting Eq. (1.1) into two equations, each of which can be solved analytically. The first of these equations is obtained by only retaining the diffusion term on the right-hand side of Eq. (1.1). This yields

$$
\frac{\partial A^{(1)}}{\partial t}=q^{2}\left(c_{0}+i\right) \nabla^{2} A^{(1)} .
$$

The second equation contains the remaining growth and nonlinear terms and is given by

$$
\frac{\partial A^{(2)}}{\partial t}=\rho A^{(2)}-(\rho-i) A^{(2)}\left|A^{(2)}\right|^{2} .
$$

The key element in this splitting is that Eqs. (2.1) and (2.2) both can be solved analytically. This fact makes it possible to solve Eq. (1.1) by an interleaving procedure which we now describe. Another important feature is that it is natural to solve Eq. (2.2) in physical space, while Eq. (2.1), given the periodic boundary conditions, is most naturally solved in Fourier space.

We now obtain the solutions to Eqs. (2.1) and (2.2), to be referred to as the diffusion and nonlinear solutions, respectively. For Eq. (2.1), the expansion

$$
A^{(1)}(\mathbf{x}, t)=\sum_{k_{1}, \ldots, k_{d}=-\infty}^{\infty} f_{\mathbf{k}}^{(1)}(t) \exp [i \mathbf{k} \cdot \mathbf{x}], \quad \mathbf{x} \in[0,2 \pi]^{d},
$$

where $\mathbf{k}=\left(k_{1}, \ldots, k_{d}\right)$, is used to reduce Eq. (2.1) to a system of differential equations for the Fourier coefficients $f_{\mathbf{k}}^{(1)}$ :

$$
\frac{d f_{\mathbf{k}}^{(1)}}{d t}=-|\mathbf{k}|^{2} q^{2}\left(c_{0}+i\right) f_{\mathbf{k}}^{(1)} \quad \forall \mathbf{k}
$$


This can be solved i nmediately, so that

$$
f_{\mathbf{k}}^{(1)}(t)=f_{\mathbf{k}}^{(1)}(0) \exp \left[-|\mathbf{k}|^{2} q^{2}\left(c_{0}+i\right) t\right] \quad \forall \mathbf{k},
$$

and substitution into the expansion (2.3) then yields the physical-space representation of the solution:

$$
A^{(1)}(\mathbf{x}, t)=\sum_{k_{1}, \ldots, k_{d}=-\infty}^{\infty} f_{\mathbf{k}}^{(1)}(0) \exp \left[-|\mathbf{k}|^{2} q^{2}\left(c_{0}+i\right) t\right] \exp [i \mathbf{k} \cdot \mathbf{x}], \quad \mathbf{x} \in[0,2 \pi]^{d}
$$

To solve Eq. (2.2), the physical coordinate $\mathbf{x}$ is regarded as a parameter, and the solution is assumed to be of the form

$$
A^{(2)}(\cdot, t)=R(\cdot, t) \exp [i \Theta(\cdot, t)]
$$

On substitution into Eq. (2.2), the real and imaginary parts yield the differential equations:

$$
\begin{aligned}
& \frac{d R}{d t}=\rho\left(R-R^{3}\right), \\
& \frac{d \Theta}{d t}=R^{2} .
\end{aligned}
$$

Integrating Eq. (2.8) and determining the constant of integration yields

$$
R(\cdot, t)=\frac{R(\cdot, 0) e^{\rho t}}{\sqrt{1-R^{2}(\cdot, 0)+R^{2}(\cdot, 0) e^{2 \rho t}}} .
$$

Next, using this result, Eq. (2.9) can be integrated to obtain

$$
\Theta(\cdot, t)=\Theta(\cdot, 0)+\frac{1}{2 \rho} \ln \left(1-R^{2}(\cdot, 0)+R^{2}(\cdot, 0) e^{2 \rho t}\right) .
$$

Hence, the solution is

$$
\begin{aligned}
A^{(2)}(\mathbf{x}, t)= & \frac{R(\mathbf{x}, 0) e^{\rho t}}{\sqrt{1-R^{2}(\mathbf{x}, 0)+R^{2}(\mathbf{x}, 0) e^{2 \rho t}}} \\
& \times \exp \left\{i\left[\Theta(\mathbf{x}, 0)+\frac{1}{2 \rho} \ln \left(1-R^{2}(\mathbf{x}, 0)+R^{2}(\mathbf{x}, 0) e^{2 \rho t}\right)\right]\right\},
\end{aligned}
$$

where $\mathbf{x} \in[0,2 \pi]^{d} . A^{(2)}$ is now taken to be a proper function of $\mathbf{x}$ and $t$ and will be written $A^{(2)}(\mathbf{x}, t)$. Similarly, the amplitude $R$ and the phase $\Theta$ will be written $R(\mathbf{x}, t)$ and $\Theta(\mathbf{x}, t)$.

Now we must combine the analytical solutions (2.6) and (2.12) to produce an overall solution of Eq. (1.1). This is done by repeatedly using $A^{(1)}$ and $A^{(2)}$ in a fixed order and matching the two solutions at each step or fractional step. For this purpose the time will be discretized into steps of size $\Delta t$.

In order to represent the two analytical solutions on a digital computer, it is also necessary to discretize the spatial variable into steps of size $\Delta x=2 \pi / N$ and to make the corresponding truncation of the Fourier spectrum, $f_{\mathbf{k}} \equiv 0$ for $\left|k_{\alpha}\right|>N / 2$, 
$\alpha=1, \ldots, d$. Using a finite Fourier transform introduces aliasing errors in the coefficients, so that

$$
f_{\mathbf{k}}=\bar{f}_{\mathbf{k}}+\sum_{m_{1}, \ldots, m_{d}=-\infty}^{\infty} \bar{f}_{\mathbf{k}+\mathbf{m} N / 2},
$$

where $\bar{f}_{\mathbf{k}}$ is the exact result. However, it will be assumed that $N$ is large enough so that the errors introduced by the spatial approximation of $A$ will be much smaller than the errors introduced by the temporal approximation, and so only the temporal accuracy of our splitting method will be shown analytically.

For what follows we define the discretized versions of Eqs. (2.6) and (2.12). We take $N$ modes in each direction in both physical and Fourier space and define

$$
\mathbf{x}_{\mathbf{j}}=2 \pi \mathbf{j} / N=\frac{2 \pi}{N}\left(j_{1}, \ldots, j_{d}\right) .
$$

The subscript $\mathbf{j}$ henceforth denotes evaluation at $\mathbf{x}_{\mathbf{j}}$. Thus, for arbitrary initial time $t_{0}$, Eqs. (2.6) and (2.12) become

$$
A_{\mathbf{j}}^{(1)}\left(t_{0}+t\right)=\sum_{k_{1}, \ldots, k_{d}=-N / 2}^{N / 2} f_{\mathbf{k}}^{(1)}\left(t_{0}\right) \exp \left[-|\mathbf{k}|^{2} q^{2}\left(c_{0}+i\right) t\right] \exp \left[i \mathbf{k} \cdot \mathbf{x}_{\mathbf{j}}\right]
$$

and

$$
\begin{aligned}
A_{\mathbf{j}}^{(2)}\left(t_{0}+t\right)= & \frac{R_{\mathbf{j}}\left(t_{0}\right) e^{\rho t}}{\sqrt{1-R_{\mathbf{j}}^{2}\left(t_{0}\right)+R_{\mathbf{j}}^{2}\left(t_{0}\right) e^{2 \rho t}}} \\
& \times \exp \left\{i\left[\Theta_{\mathbf{j}}\left(t_{0}\right)+\frac{1}{2 \rho} \ln \left(1-R_{\mathbf{j}}^{2}\left(t_{0}\right)+R_{\mathbf{j}}^{2}\left(t_{0}\right) e^{2 \rho t}\right)\right\}\right\} .
\end{aligned}
$$

\section{Combined solutions-analytical splitting schemes.}

3.1. First-order accuracy. The most obvious way to combine solutions (2.6) and (2.12) is simply to alternate them; first using $A^{(1)}$ to advance the solution $\Delta i$, then using $A^{(2)}$ to advance the result $\Delta t$, and so on. Using only $A^{(1)}$ for time $\Delta i$ may be termed a half step because only "half" of the dynamics of Ea. (1.1) is included. This first step produces an intermediate, nonphysical result at a time that we wil: denote as $t_{*}$. The second step, using $A^{(2)}$ for time $\Delta t$, then gives an approximation of the solution of Eq. (1.1) after one full time step. We now show that this method is first-order accurate and discuss its implementation.

If we assume the same $N$-point spatial discretization in all directions, then Eq (1.1) becomes

$$
\frac{d A_{\mathbf{j}}}{d t}=F_{\mathbf{j}}(\mathbf{A})+G_{\mathbf{j}}(\mathbf{A})
$$

where

$$
F_{\mathbf{j}}(\mathbf{A})=\frac{q^{2}\left(c_{0}+i\right)}{N} \sum_{k_{1}, \ldots, k_{d}=-N / 2}^{N / 2} \sum_{m_{1}, \ldots, m_{d}=0}^{N-1}-|\mathbf{k}|^{2} A_{\mathbf{m}} \exp \left[i \mathbf{k} \cdot \mathbf{x}_{\mathbf{j}}\right] \exp \left[-i \mathbf{k} \cdot \mathbf{x}_{\mathbf{m}}\right]
$$

and

$$
G_{\mathbf{j}}(\mathbf{A})=\rho A_{\mathbf{j}}+(-\rho+i) A_{\mathbf{j}}\left|A_{\mathbf{j}}\right|^{2} .
$$


Here A denotes the $N^{d}$-component vector consisting of the values of $A$ at all the spatial grid points. Similarly, f will denote the $N^{d}$-component vector of Fourier coefficients. It can be shown that the discretized solutions $(2.15),(2.16)$ satisfy the equations

$$
\begin{aligned}
& \frac{d A_{\mathbf{j}}^{(1)}}{d t}=F_{\mathbf{j}}(\mathbf{A}), \\
& \frac{d A_{\mathbf{j}}^{(2)}}{d t}=G_{\mathbf{j}}(\mathbf{A}) .
\end{aligned}
$$

Given the above framework of Eqs. (3.4), (3.5) and their solutions, the accuracy of the combined solution of Eq. (3.1) can now be derived. Because the temporal accuracy of this splitting method is the same for all $N$ in Eqs. (3.1)-(3.5), only the one-mode $(N=1)$ case will be treated and hence all subscripts will be dropped.

Denoting the exact solution to Eq. (3.1) as $A$, a Taylor expansion yields

$$
A(t+\Delta t)=A(t)+\Delta t \dot{A}(t)+\frac{\Delta t^{2}}{2} \ddot{A}(t)+O\left(\Delta t^{3}\right),
$$

where ${ }^{\cdot}=d / d t$. Using Eq. (3.1), this gives

$$
\begin{aligned}
A(t+\Delta t)= & A(t)+\Delta t[F(A(t))+G(A(t))]+\frac{\Delta t^{2}}{2}\left[F^{\prime}(A(t))+G^{\prime}(A(t))\right] \\
& \times[F(A(t))+G(A(t))]+O\left(\Delta t^{3}\right)
\end{aligned}
$$

for the exact solution at time $t+\Delta t$, where ${ }^{\prime}=\partial / \partial A$.

First applying the solution to Eq. (3.4), the splitting solution of Eq. (3.1) after the first half step is

$$
\widetilde{A}\left(t_{*}\right)=A^{(1)}(t+\Delta t)=A^{(1)}(t)+\Delta t \dot{A}^{(1)}(t)+\frac{\Delta t^{2}}{2} \ddot{A}^{(1)}(t)+O\left(\Delta t^{3}\right) .
$$

Substituting $A^{(1)}(t)=A(t)$ and using Eq. (3.4) gives

$$
\widetilde{A}\left(t_{*}\right)=A(t)+\Delta t F(A(t))+\frac{\Delta t^{2}}{2} F^{\prime}(A(t)) F(A(t))+O\left(\Delta t^{3}\right) .
$$

The second half step, using the solution to Eq. (3.5), gives

$$
\begin{aligned}
\widetilde{A}(t+\Delta t) & =A^{(2)}\left(t_{*}+\Delta t\right) \\
& =A^{(2)}\left(t_{*}\right)+\Delta t \dot{A}^{(2)}\left(t_{*}\right)+\frac{\Delta t^{2}}{2} \ddot{A}^{(2)}\left(t_{*}\right)+O\left(\Delta t^{3}\right) .
\end{aligned}
$$

Therefore,

$$
\begin{aligned}
\widetilde{A}(t+\Delta t)= & A(t)+\Delta t F(A(t))+\frac{\Delta t^{2}}{2} F^{\prime}(A(t)) F(A(t)) \\
& +\Delta t G\left(\widetilde{A}\left(t_{*}\right)\right)+\frac{\Delta t^{2}}{2} G^{\prime}\left(\widetilde{A}\left(t_{*}\right)\right) G\left(\widetilde{A}\left(t_{*}\right)\right)+O\left(\Delta t^{3}\right),
\end{aligned}
$$


where we have used Eq. (3.5) and the fact that $A^{(2)}\left(t_{*}\right)=\widetilde{A}\left(t_{*}\right)$. Taylor expanding the $G$ and $G^{\prime}$ terms in the above relation about $A(t)$ finally yields

$$
\begin{aligned}
\widetilde{A}(t+\Delta t)= & A(t)+\Delta t[F(A(t))+G(A(t))] \\
& +\frac{\Delta t^{2}}{2}\left[F^{\prime}(A(t)) F(A(t))+G^{\prime}(A(t)) G(A(t))\right] \\
& +\Delta t^{2} G^{\prime}(A(t)) F(A(t))+O\left(\Delta t^{3}\right) .
\end{aligned}
$$

Using the exact expansion derived above, Eq. (3.12) can be rewritten as

$$
\widetilde{A}(t+\Delta t)=(3.7)+\frac{\Delta t^{2}}{2}\left[G^{\prime} F-F^{\prime} G\right]+O\left(\Delta t^{3}\right) .
$$

Therefore, the error in the approximate method described above is $O\left(\Delta t^{2}\right)$ per time step, which implies that this scheme is first-order accurate in time.

REMARK 1. It can be shown that when the solutions to Eqs. (3.4) and (3.5) are applied in the order opposite to that used to derive Eq. (3.13), the error is $\frac{1}{2} \Delta t^{2}\left[F^{\prime} G-\right.$ $G^{\prime} F$ ]. Therefore, this method is also first-order accurate.

REMARK 2. We note that the first-order accuracy shown above is true for the general case of equations of the form (3.1) having splittings, such as Eqs. (3.4) and (3.5), which can be solved analytically.

3.2. Implementation of first-order scheme. To describe the details of applying our splitting methods to Eq. (1.1), we define the step-wise solutions to Eqs. (3.4) and (3.5). Replacing $t$ by $\Delta t$ and $t_{0}$ by $t$, Eqs. (2.15) and (2.16) become

$$
A_{\mathbf{j}}^{(1)}(t+\Delta t)=\sum_{k_{1}, \ldots, k_{d}=-N / 2}^{N / 2} f_{\mathbf{k}}(t) \exp \left[-|\mathbf{k}|^{2} q^{2}\left(c_{0}+i\right) \Delta t\right] \exp \left[i \mathbf{k} \cdot \mathbf{x}_{\mathbf{j}}\right]
$$

and

$$
\begin{aligned}
A_{\mathrm{j}}^{(2)}(t+\Delta t)= & \frac{R_{\mathbf{j}}(t) e^{\rho \Delta t}}{\sqrt{1-R_{\mathbf{j}}^{2}(t)+R_{\mathbf{j}}^{2}(t) e^{2 \rho \Delta t}}} \\
& \times \exp \left\{i\left[\Theta_{\mathbf{j}}(t)+\frac{1}{2 \rho} \ln \left(1-R_{\mathbf{j}}^{2}(t)+R_{\mathbf{j}}^{2}(t) e^{2 \rho \Delta t}\right)\right]\right\} .
\end{aligned}
$$

Solution operators corresponding to Eqs. (3.14) and (3.15) are now defined as

$$
\begin{aligned}
& \mathscr{S}_{D}(\Delta t): \mathbf{A}(t) \rightarrow \mathbf{A}^{(1)}(t+\Delta t), \\
& \mathscr{S}_{N}(\Delta t): \mathbf{A}(t) \rightarrow \mathbf{A}^{(2)}(t+\Delta t) .
\end{aligned}
$$

The above operators act by taking the diffusion (or nonlinear) solution at time $t$ to be the initial condition, i.e., by setting $\mathbf{A}^{(1)}(t)=\mathbf{A}(t)$ (or $\left.\mathbf{A}^{(2)}(t)=\mathbf{A}(t)\right)$.

The first-order splitting solution to Eq. (1.1) can now be represented as

$$
\widetilde{\mathbf{A}}\left(t_{n+1}\right)=\mathscr{S}_{N} \circ \mathscr{S}_{D} \widetilde{\mathbf{A}}\left(t_{n}\right),
$$


where $t_{n}=n \Delta t$ and $\widetilde{\mathbf{A}}(0)=\mathbf{A}(0)$. This compact description of the splitting method hides the important fact that the amount of computational work required for the application of $\mathscr{S}_{D}$ is much greater than for $\mathscr{S}_{N}$, since the calculation is done in physical space and the diffusion equation (3.4) is actually solved in Fourier space.

The actual computer implementation of this first-order splitting method can be most clearly described by defining the operator corresponding to the step-wise, discretized version of Eq. (2.5), the diffusion solution in Fourier space. This operator, requiring $O\left(N^{d}\right)$ operations, is

$$
\widehat{\mathscr{S}}_{D}(\Delta t): \mathbf{f}(t) \rightarrow \mathbf{f}^{(1)}(t+\Delta t) .
$$

As with $\mathscr{S}_{N}$ and $\mathscr{S}_{D}, \widehat{\mathscr{S}}_{D}$ is applied using the initial condition $\mathbf{f}^{(1)}(t)=\mathbf{f}(t)$. Taking $\mathscr{F}$ to be the $d$-dimensional complex FFT, the approximate solution is then

$$
\widetilde{\mathbf{A}}\left(t_{n+1}\right)=\mathscr{S}_{N} \circ \mathscr{F}^{-1} \circ \widehat{\mathscr{S}}_{D} \circ \mathscr{F} \widetilde{\mathbf{A}}\left(t_{n}\right),
$$

since in practice

$$
\mathscr{S}_{D}=\mathscr{F}^{-1} \circ \widehat{\mathscr{S}}_{D} \circ \mathscr{F} .
$$

This first-order scheme for Eq. (1.1) requires two $O\left([N \log N]^{d}\right)$ FFTs per time step, as does the first-order scheme

$$
\widetilde{\mathbf{A}}\left(t_{n+1}\right)=\mathscr{F}^{-1} \circ \widehat{\mathscr{S}}_{D} \circ \mathscr{F} \circ \mathscr{S}_{N} \tilde{\mathbf{A}}\left(t_{n}\right) .
$$

Finally, in Fourier space, the two solutions become

$$
\tilde{\mathbf{f}}\left(t_{n+1}\right)=\mathscr{F} \circ \mathscr{S}_{N} \circ \mathscr{F}^{-1} \circ \widehat{\mathscr{S}_{D}} \tilde{\mathbf{f}}\left(t_{n}\right)
$$

and

$$
\tilde{\mathbf{f}}\left(t_{n+1}\right)=\widehat{\mathscr{S}}_{D} \circ \mathscr{F} \circ \mathscr{S}_{N} \circ \mathscr{F}^{-1} \tilde{\mathbf{f}}\left(t_{n}\right),
$$

using $\tilde{\mathbf{f}}\left(t_{n}\right)=\mathscr{F} \widetilde{\mathbf{A}}\left(t_{n}\right)$. It should be noted that Eqs. (3.22) and (3.23) give $A$ in both physical and Fourier spaces at each step without requiring an additional FFT.

3.3. Second-order accuracy. In the numerical solution of a partial differential equation, when it is desirable to have good spatial and temporal accuracy as well as to observe the solution over an extended period of time, a first-order method is often not sufficient. This was the case for the regimes of interest in our study of the CGL equation (1.1). Fortunately, as we now show, the first-order splitting scheme described above can be turned into a second-order method requiring only slightly more computational work per time step.

As described above, the $O\left(\Delta t^{2}\right)$ error terms in the approximate solution (3.12) change only in sign upon reversal of the order in which $A^{(1)}$ and $A^{(2)}$ are used. This suggests that alternating the order in which $\mathscr{S}_{D}$ and $\mathscr{S}_{N}$ are applied from one time step to the next might result in the cancellation of the $O\left(\Delta t^{2}\right)$ terms over two time steps . 
We advance (3.12) $\Delta t$ using $A^{(2)}$, Taylor expand about $t$, and then advance the result another $\Delta t$ using $A^{(1)}$. Taylor expanding this result yields

$$
\begin{aligned}
\tilde{A}(t+2 \Delta t)= & A(t)+2 \Delta t[F+G] \\
& +\frac{4 \Delta t^{2}}{2}\left[F^{\prime} F+G^{\prime} G+G^{\prime} F+G F^{\prime}\right]+O\left(\Delta t^{3}\right),
\end{aligned}
$$

the argument $A(t)$ being implied in $F, F^{\prime}, G$, and $G^{\prime}$.

A comparison of Eq. (3.25) with the exact expansion (3.7) shows that the overall error of this two-step scheme is $O\left(\Delta t^{3}\right)$ (the form of the error terms of $O\left(\Delta t^{3}\right)$ are not shown in Eq. (3.25)). Replacing $\Delta t$ by $\Delta t / 2$ means the splitting solution

$$
\widetilde{\mathbf{A}}\left(t_{n+1}\right)=\mathscr{S}_{D}(\Delta t / 2) \circ \mathscr{S}_{N}(\Delta t / 2) \circ \mathscr{S}_{N}(\Delta t / 2) \circ \mathscr{S}_{D}(\Delta t / 2) \tilde{\mathbf{A}}\left(t_{n}\right)
$$

is second-order accurate in time, as is the solution

$$
\widetilde{\mathbf{A}}\left(t_{n+1}\right)=\mathscr{S}_{N}(\Delta t / 2) \circ \mathscr{S}_{D}(\Delta t / 2) \circ \mathscr{S}_{D}(\Delta t / 2) \circ \mathscr{S}_{N}(\Delta t / 2) \widetilde{\mathbf{A}}\left(t_{n}\right) .
$$

Remark. Again, the schemes (3.26) and (3.27) are also second-order accurate when applied to any p.d.e. that can be split into two analytically solvable parts.

3.4. Implementation of second-order scheme. Since our solutions to the split equations (3.4) and (3.5) are exact, both solution operators have the semigroup property, $\mathscr{S}(\Delta t / 2) \circ \mathscr{S}(\Delta t / 2)=\mathscr{S}(\Delta t)$. Thus, the second-order schemes (3.26) and (3.27) can be simplified to

$$
\tilde{\mathbf{A}}\left(t_{n+1}\right)=\mathscr{S}_{D}(\Delta t / 2) \circ \mathscr{S}_{N}(\Delta t) \circ \mathscr{S}_{D}(\Delta t / 2) \tilde{\mathbf{A}}\left(t_{n}\right)
$$

and

$$
\widetilde{\mathbf{A}}\left(t_{n+1}\right)=\mathscr{S}_{N}(\Delta t / 2) \circ \mathscr{S}_{D}(\Delta t) \circ \mathscr{S}_{N}(\Delta t / 2) \tilde{\mathbf{A}}\left(t_{n}\right)
$$

Now, as in the first-order case, we use the relation (3.21), and so Eqs. (3.28) and (3.29) become

$$
\widetilde{\mathbf{A}}\left(t_{n+1}\right)=\mathscr{F}^{-1} \circ \widehat{\mathscr{S}}_{D}(\Delta t / 2) \circ \mathscr{F} \circ \mathscr{S}_{N}(\Delta t) \circ \mathscr{F}^{-1} \circ \widehat{\mathscr{S}}_{D}(\Delta t / 2) \circ \mathscr{F} \widetilde{\mathbf{A}}\left(t_{n}\right)
$$

and

$$
\tilde{\mathbf{A}}\left(t_{n+1}\right)=\mathscr{S}_{N}(\Delta t / 2) \circ \mathscr{F}^{-1} \circ \widehat{\mathscr{S}}_{D}(\Delta t) \circ \mathscr{F} \circ \mathscr{S}_{N}(\Delta t / 2) \tilde{\mathbf{A}}\left(t_{n}\right) .
$$

If only the physical-space representation of $A$ is required at each $t_{n}$, then Eq. (3.31) can be used, giving a second-order method that requires only one more $O\left(N^{d}\right)$ procedure per time step than the first-order method. Similarly, applying $\mathscr{F}$ to both sides of Eq. (3.30) yields the most efficient second-order splitting scheme in Fourier space:

$$
\tilde{\mathbf{f}}\left(t_{n+1}\right)=\widehat{\mathscr{S}}_{D}(\Delta t / 2) \circ \mathscr{F} \circ \mathscr{S}_{N}(\Delta t) \circ \mathscr{F}^{-1} \circ \widehat{\mathscr{S}}_{D}(\Delta t / 2) \tilde{\mathbf{f}}\left(t_{n}\right)
$$

If the solution is required in both physical and Fourier spaces at each $t_{n}$, then Eq. (3.31) or (3.32) should be used, with a third FFT per step being added to obtain the desired representation of the data.

Thus, even in the worst case, the second-order splitting method described above only requires one more $O\left(N^{d}\right)$ application of either $\mathscr{S}_{N}$ or $\widehat{\mathscr{S}}_{D}$ and one more 
$O\left([N \log N]^{d}\right)$ FFT per step than does the first-order method. The efficiency of the second-order splitting method makes it far superior to the first-order version. The schemes (3.31) and (3.32) are also much simpler to implement and more efficient than second-order spectral or pseudospectral schemes for solving Eq. (1.1). For these reasons, our numerical studies of the CGL equation were performed using the secondorder splitting method (3.32).

4. Validation of second-order method. We now verify numerically that the splitting method (3.32) is second-order accurate in time and gives spectral accuracy in space. We do this by applying the method (3.32) to both the one-dimensional and twodimensional versions of Eq. (1.1). We also show that these schemes correctly solve Eq. (1.1) by direct comparison with known results.

In performing the following tests of the scheme (3.32), the two-dimensional CGL equation was solved using fully periodic boundary conditions in both spatial directions. However, all one-dimensional calculations were done in the invariant subspace of the odd periodic solutions, using

$$
A_{j}^{(1)}(t)=\sum_{k=1}^{N / 2-1} f_{k}(t) \sin k x_{j}
$$

instead of the general form (2.3). This form of the solution can be obtained by using the complex $N$-point FFT as described above and then setting $f_{k}^{\prime}=\left(f_{k}-f_{-k}\right) / 2$ and $f_{-k}^{\prime}=-f_{k}^{\prime}$ after each application of $\mathscr{F}$. For our calculations, we used two $(N / 2-1)$-point real sine transforms. Using only the sine modes, which is equivalent to enforcing Dirichlet boundary conditions, allowed us to compare our one-dimensional results with those obtained by Sirovich, Rodriguez, and Knight [15].

4.1. Convergence. The first tests performed on the splitting method involved checking that it converged properly as the step sizes in time and space were decreased. For the one-dimensional case we used the parameters $q=0.1$ and $\rho=c_{0}=0.25$ and the initial condition $A_{j}(0)=.02(1+i) \sin x_{j}$. For the two-dimensional case, we used the parameters $q=\sqrt{0.45}$ and $\rho=c_{0}=1 / \sqrt{3}$ and the initial condition $f_{k l}(0)=0.01$ for $k^{2}+l^{2} \leq 1$.

To test temporal convergence, the approximate solution was computed at a fixed time $T$ using various time steps $\Delta t$. For the one-dimensional case, spatial accuracy was kept high by taking $N=256$. The measure of the difference between two solutions was taken to be their root mean square difference at the grid points:

$$
d\left[\tilde{A}_{j}(T ; \Delta t), \tilde{A}_{j}\left(T ; \Delta t^{\prime}\right)\right]=\sqrt{\frac{1}{N} \sum_{k=1}^{N / 2-1}\left|\tilde{A}_{j}(T ; \Delta t)-\tilde{A}_{j}\left(T ; \Delta t^{\prime}\right)\right|^{2}} .
$$

Given the exact solution $A_{j}(T)$, the error in the solution at time $T$ obtained using step size $\Delta t$ is then $e(\Delta t)=d\left[\tilde{A}_{j}(T ; \Delta t), A_{j}(T)\right]$. However, since $A_{j}(T)$ is not known for the CGL equation, we took the solution obtained using a very small $\Delta t$ to be the exact solution at time $T$. Deviations from this solution for larger step 


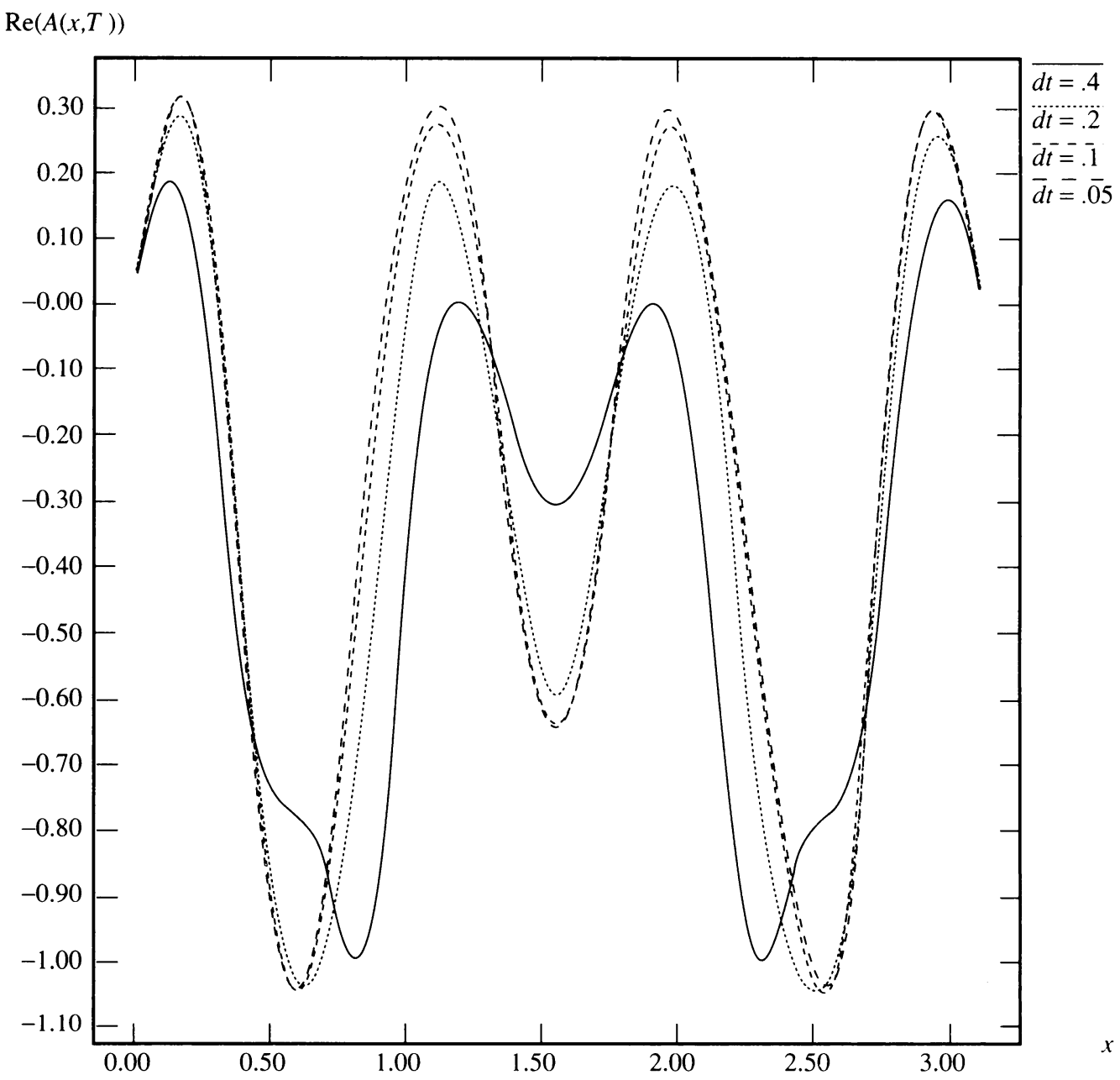

FIG. 4.1. Point-wise temporal convergence of $A$ computed by the second-order splitting method for the one-dimensional CGL equation. We show the real part of $A(x, T)$ for various step sizes.

sizes were calculated and compared with the second-order accuracy relation $e \simeq$ $\alpha \Delta t^{2}$. These results are shown in Table 4.1. The temporal convergence for the onedimensional case is also shown graphically in Fig. 4.1.

To test the temporal convergence of our two-dimensional scheme, we used the generalization of the one-dimensional error measure defined above. These results are shown in Table 4.2. The above results show that the splitting method (3.32) exhibits the expected second-order convergence in time.

The spatial convergence of our method was tested for the same parameter sets as above, but to minimize temporal errors we used a smaller final time and a very small, fixed time step. Because the number of grid points was variable, the measure of the difference between two solutions was taken to be the difference between the values 
TABLE 4.1. Results of the test done on the temporal convergence of our second-order splitting method for the one-dimensional CGL equation.

\begin{tabular}{|c|c|c|}
\hline$\Delta t$ & $e(\Delta t)=d[A(\Delta t), A(1 \cdot e-4)]$ & $(\Delta t)^{-2} \cdot e(\Delta t)$ \\
\hline $4.00 e-1$ & $5.194 e-1$ & 3.247 \\
$2.00 e-1$ & $1.549 e-1$ & 3.873 \\
$1.00 e-1$ & $3.918 e-2$ & 3.918 \\
$5.00 e-2$ & $9.785 e-3$ & 3.914 \\
$2.50 e-2$ & $2.445 e-3$ & 3.912 \\
$1.25 e-2$ & $6.111 e-4$ & 3.911 \\
$6.25 e-3$ & $1.527 e-4$ & 3.910 \\
$3.125 e-3$ & $3.816 e-5$ & 3.908 \\
$2.00 e-3$ & $1.560 e-5$ & 3.901 \\
$1.00 e-3$ & $3.869 e-6$ & 3.869 \\
\hline
\end{tabular}

TABLE 4.2. Results of temporal convergence test of second-order splitting method for the two-dimensional CGL equation. The data shown was obtained using $N=32$ grid points in each direction and the final time $T=20$.

\begin{tabular}{|l|c|c|c|}
\hline \multicolumn{1}{|c|}{$\Delta t$} & $d[A(\Delta t), A(.001)]$ & $e(\Delta t)=d[A(\Delta t), A(\Delta t / 2)]$ & $(\Delta t)^{-2} \cdot e(\Delta t)$ \\
\hline 0.1 & $6.196 e-2$ & $4.695 e-2$ & 4.695 \\
0.05 & $1.504 e-2$ & $1.131 e-2$ & 4.525 \\
0.025 & $3.724 e-3$ & $2.799 e-3$ & 4.479 \\
0.0125 & $9.246 e-4$ & $6.981 e-4$ & 4.468 \\
0.00625 & $2.266 e-4$ & - & - \\
0.002 & $1.785 e-5$ & $1.785 e-5$ & 4.464 \\
\hline
\end{tabular}

they gave for the integral of $|A|^{2}$. With the definition

$$
D\left[\tilde{A}_{j}(T ; N), \tilde{A}_{j}\left(T ; N^{\prime}\right)\right]=\left.\left|\frac{2}{N} \sum_{j=1}^{N / 2}\right| \tilde{A}_{j}(T ; N)\right|^{2}-\frac{2}{N^{\prime}} \sum_{j=1}^{N^{\prime} / 2}\left|\tilde{A}_{j}\left(T ; N^{\prime}\right)\right|^{2} \mid
$$

for the one-dimensional case, the error is $E(N)=D\left[\tilde{A}_{j}(T ; N), A_{j}\right]$, where as before $A_{j}$ is the exact solution.

Since again $A_{j}$ was not known, the values for the errors were actually obtained by evaluating $D\left[\tilde{A}_{j}, \tilde{A}_{j}^{\prime}\right]$ for $N<N^{\prime} / 2$, with $N^{\prime}$ large and fixed. For an algebraically convergent method, $E(N) \sim N^{-p}$ for some $p>0$, which implies that the ratio $E(N) / E(2 N) \sim 2^{p}$ should be approximately constant for all $N$. Table 4.3 (see p. 327), containing the results for our one-dimensional scheme, shows the errors for various $N$ 's and the ratios $E(N) / E(2 N)$. Since $E(N) / E(2 N)$ increases significantly with $N$, the method (3.32) is seen to converge faster than any power of $N$. As Fig. 4.2 shows (see p. 326), the error decreases faster than $e^{-N}$ but slower than $e^{-N^{2}}$.

To test the spatial convergence of our two-dimensional scheme, we again took a result obtained for large $N$ to be the exact solution and used as the error measure the difference between the values given for the sum over the grid points of $|A|^{2}$. As 


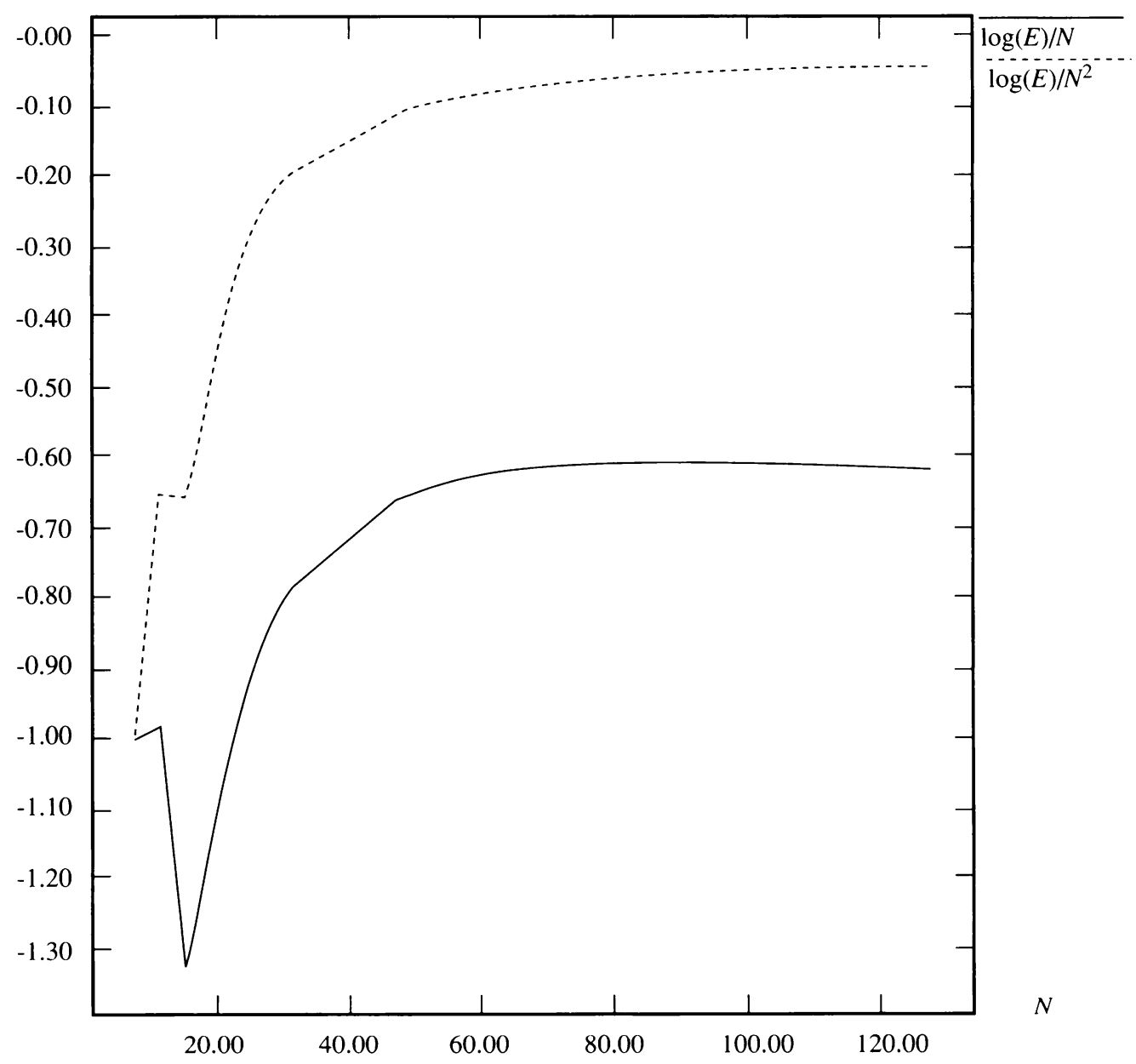

FIG. 4.2. Rate of spatial convergence of second-order splitting method for the one-dimensional CGL equation. We plot $N^{-1} \log E(N)$ and $N^{-2} \log E(N)$ vs. $N$, where these functions are normalized by their absolute values at $N=8$.

can be seen from the results in Table 4.4, our two-dimensional scheme exhibited the same exponential convergence in space as was found for the one-dimensional scheme. These results show that our second-order splitting method (3.32) converges spatially in the same way as do standard spectral methods.

4.2. Direct comparisons. The second series of experimental tests performed on the splitting method involved direct comparison with known results. Since different types of tests were done for the one-dimensional and two-dimensional cases, we treat them separately.

4.2.1. One-dimensional scheme. Using our second-order splitting scheme restricted to the subspace of the sine modes, we investigated the bifurcation sequence that occurs as $q$ is decreased for $\rho=c_{0}=0.25$. All our results, including frequency spectra and bifurcation points, were found to be in very close agreement with those described by Sirovich, Rodriguez, and Knight [15]. This fact indicates that our onedimensional splitting scheme is correctly solving the CGL equation (1.1). 
TABLE 4.3. Results of test of spatial convergence of our second-order splitting method for the one-dimensional CGL equation.

\begin{tabular}{|r|c|c|}
\hline$N$ & $E(N)=D[A(N), A(256)]$ & $E(N) / E(2 N)$ \\
\hline 16 & $1.941 e-3$ & 3.338 \\
24 & $1.140 e-3$ & 12.95 \\
28 & $8.187 e-4$ & 29.05 \\
32 & $5.814 e-4$ & 69.67 \\
40 & $2.465 e-4$ & 394.7 \\
48 & $8.806 e-5$ & 2163. \\
56 & $2.818 e-5$ & - \\
64 & $8.345 e-6$ & 87110. \\
80 & $6.245 e-7$ & - \\
96 & $4.071 e-8$ & - \\
128 & $9.58 e-11$ & - \\
\hline
\end{tabular}

TABLE 4.4. Results of spatial convergence test of second-order splitting method for the two-dimensional CGL equation. The data shown was obtained using the step size $\Delta t=10^{-4}$ and the final time $T=5$.

\begin{tabular}{|r|c|c|}
\hline$N$ & $E(N)=D[A(N), A(32)]$ & $\ln E(N) / \ln N$ \\
\hline 4 & $5.01 e-3$ & -3.82 \\
6 & $2.19 e-4$ & -4.70 \\
8 & $3.17 e-6$ & -6.09 \\
12 & $1.10 e-9$ & -8.30 \\
16 & $2.42 e-11$ & -8.82 \\
\hline
\end{tabular}

Since the parameter region of interest in our study was $\rho, c_{0} \leq 0.25$ and $q \leq 0.2$, a test was performed for the case $\rho=c_{0}=0.25, q=0.14$. This was done using the averaged wavenumber spectrum. As Fig. 4.3 shows (see p. 328), the spectrum obtained with our second-order splitting scheme is nearly identical to that calculated with the pseudospectral method used by Sirovich, Rodriguez, and Knight [15]. ${ }^{1}$ Thus the form of the splitting method (3.32) used here has been shown to approximate correctly the solution to Eq. (1.1) over a substantial range of $q$. This fact, combined with the scheme's demonstrated convergence properties, implies that, given sufficiently fine spatial and temporal discretizations, our splitting scheme will correctly solve the one-dimensional CGL equation for any parameter set of interest.

4.2.2. Two-dimensional scheme. The first test performed on our two-dimensional splitting scheme for Eq. (1.1) was based on linear stability theory, which shows that the criterion for the $A(x, y, t)=e^{i t}$ solution to be unstable to the Fourier mode

\footnotetext{
${ }^{1}$ J. D. Rodriguez provided the authors with the pseudospectral code which, after slight modification, was used to obtain the curve labeled (P-S) in Fig. 4.3. After further modifications, this code was also used for the time comparisons shown in Table 5.1.
} 
$\log F(k)$

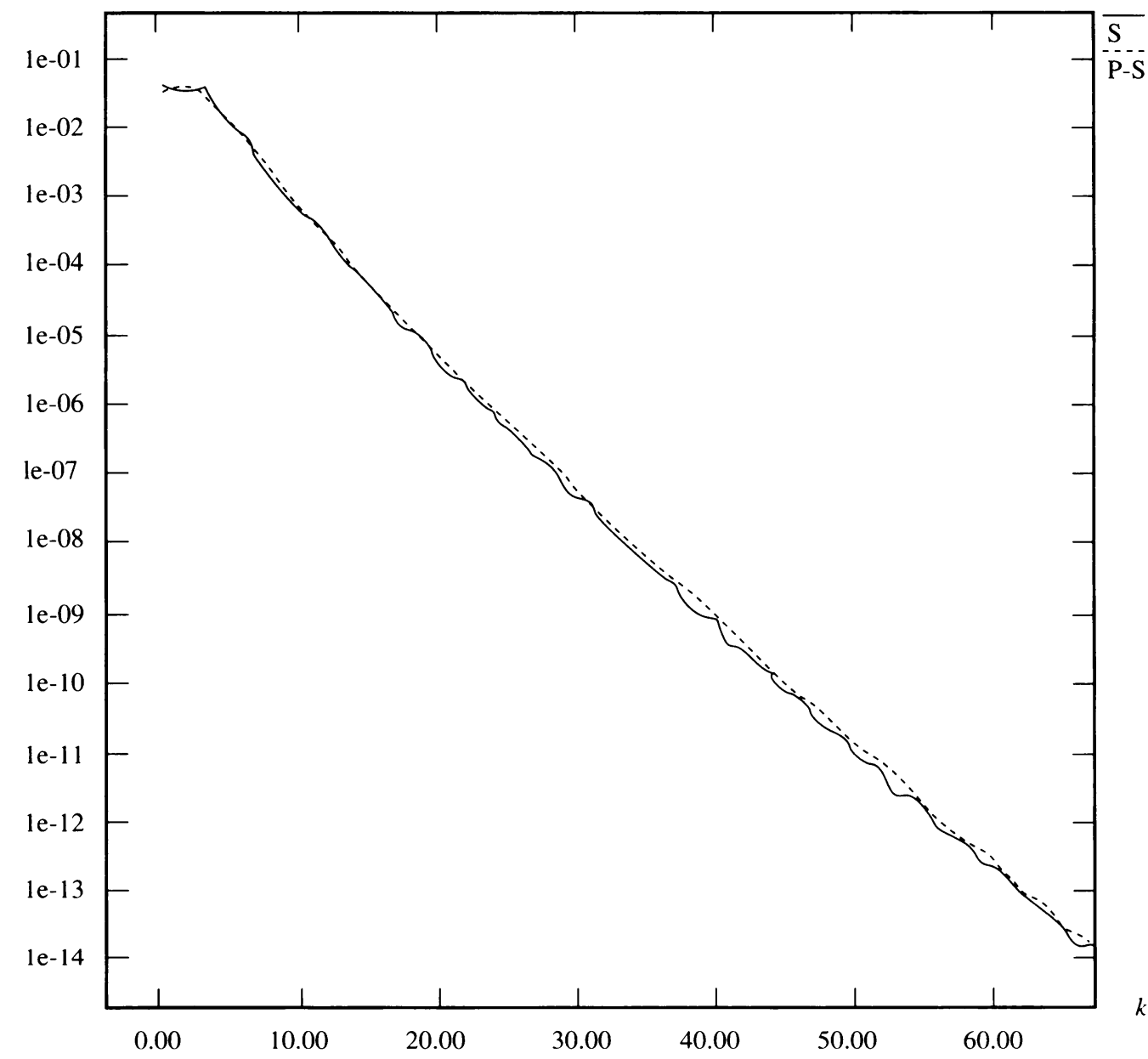

FIG. 4.3. Averaged wavenumber spectra for the one-dimensional CGL equation obtained using a pseudospectral method (P-S) and our second-order splitting method $(\mathrm{S})$. We plot $\log F(k)$ vs. $k$, where $F(k)=\left\langle\left|f_{k}\right|^{2}\right\rangle$.

$e^{i k x} e^{i l y}$ is

$$
k^{2}+l^{2}<\frac{2\left(1-\rho c_{0}\right)}{q^{2}\left(1+c_{0}^{2}\right)} .
$$

When this instability condition holds, the $(k, l)$ mode should grow from a small amplitude like $e^{\lambda_{+} t}$, where

$$
\lambda_{+}=-\left(w^{2} c_{0}+\rho\right)+\sqrt{\left(w^{2} c_{0}+\rho\right)^{2}-w^{2}\left[w^{2}\left(1+c_{0}^{2}\right)+2\left(\rho c_{0}-1\right)\right]}
$$

and $w^{2}=q^{2}\left(k^{2}+l^{2}\right)$.

These linear stability results were used to test our two-dimensional splitting scheme near the first bifurcation, which corresponds to $q=1$ for $\rho=c_{0}=1 / \sqrt{3}$. The initial condition used for investigating the stability of the Stokes solution to the Fourier 
$F(k, l, t) \times 10^{-6}$

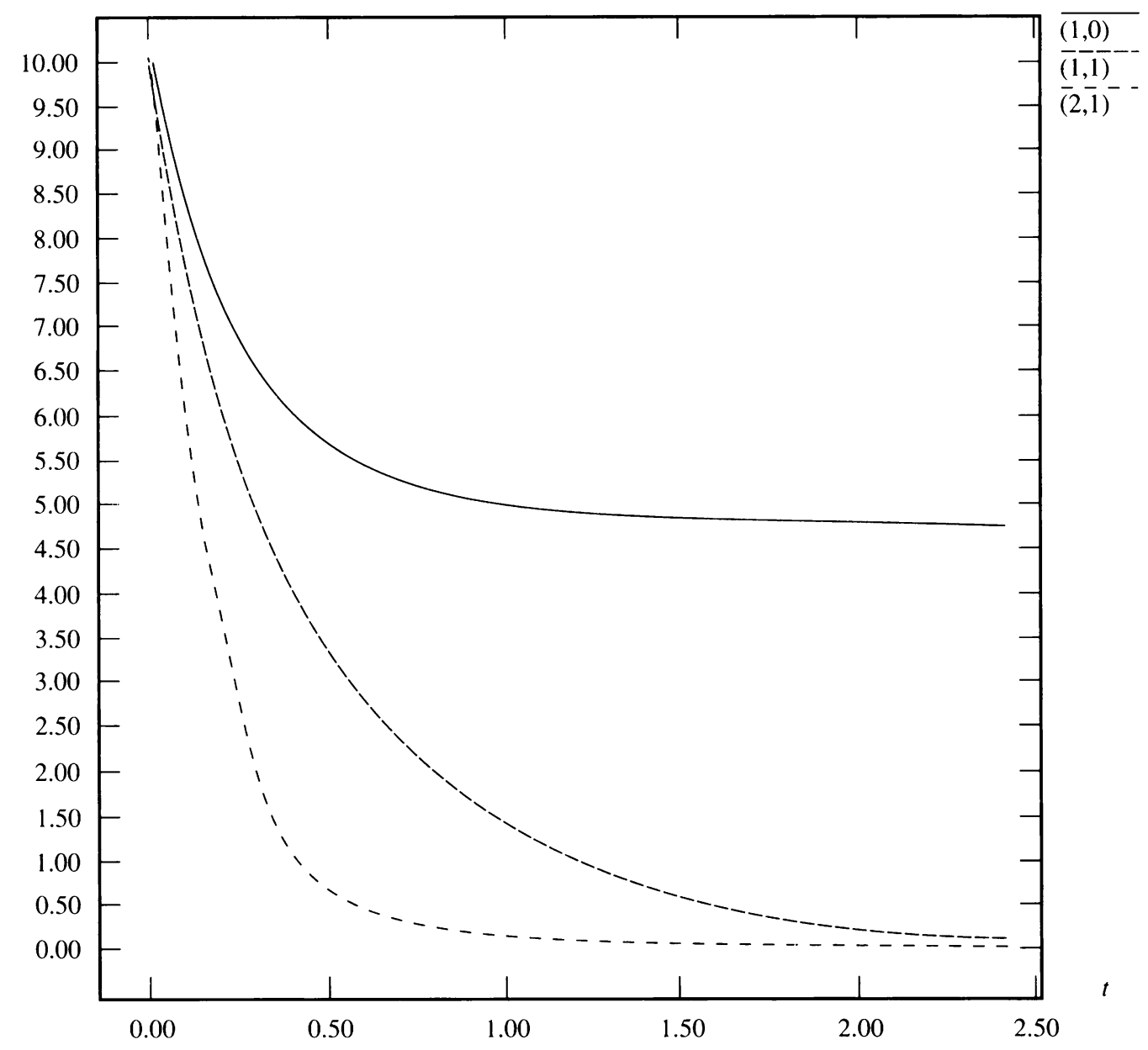

Fig. 4.4. Agreement of results obtained using our two-dimensional splitting scheme with the linear stability prediction for $q=1.01$. We plot $F(k, l, t)$ vs. $t$ for the modes $(1,0),(1,1)$, and $(2,1)$, where $F(k, l, t)=\left|f_{k l}(t)\right|$.

mode $(k, l)$ was $f_{00}(t=0)=1, f_{k l}(t=0)=10^{-5}$. For $q>1$, the magnitudes of all Fourier coefficients except $f_{00}$ were found to decrease asymptotically to zero, as expected. This behavior for $q=1.01$ is shown in Fig. 4.4. For $q$ slightly less than 1 , only the modes $( \pm 1,0)$ and $(0, \pm 1)$ grew from small initial amplitudes, which is also what was expected from the linear analysis. Figure 4.5 (see p. 330) shows this for the case $q=0.9$.

The rate of growth of the linearly unstable modes given by our two-dimensional scheme was measured for several values of $q$ and found to be very close to $\lambda_{+}$given by the above formula. These results are shown in Table 4.5 (see p. 330). These tests indicate that our splitting scheme is giving the correct behavior of solutions of the two-dimensional CGL equation. 
$\log F(k, l, t)$

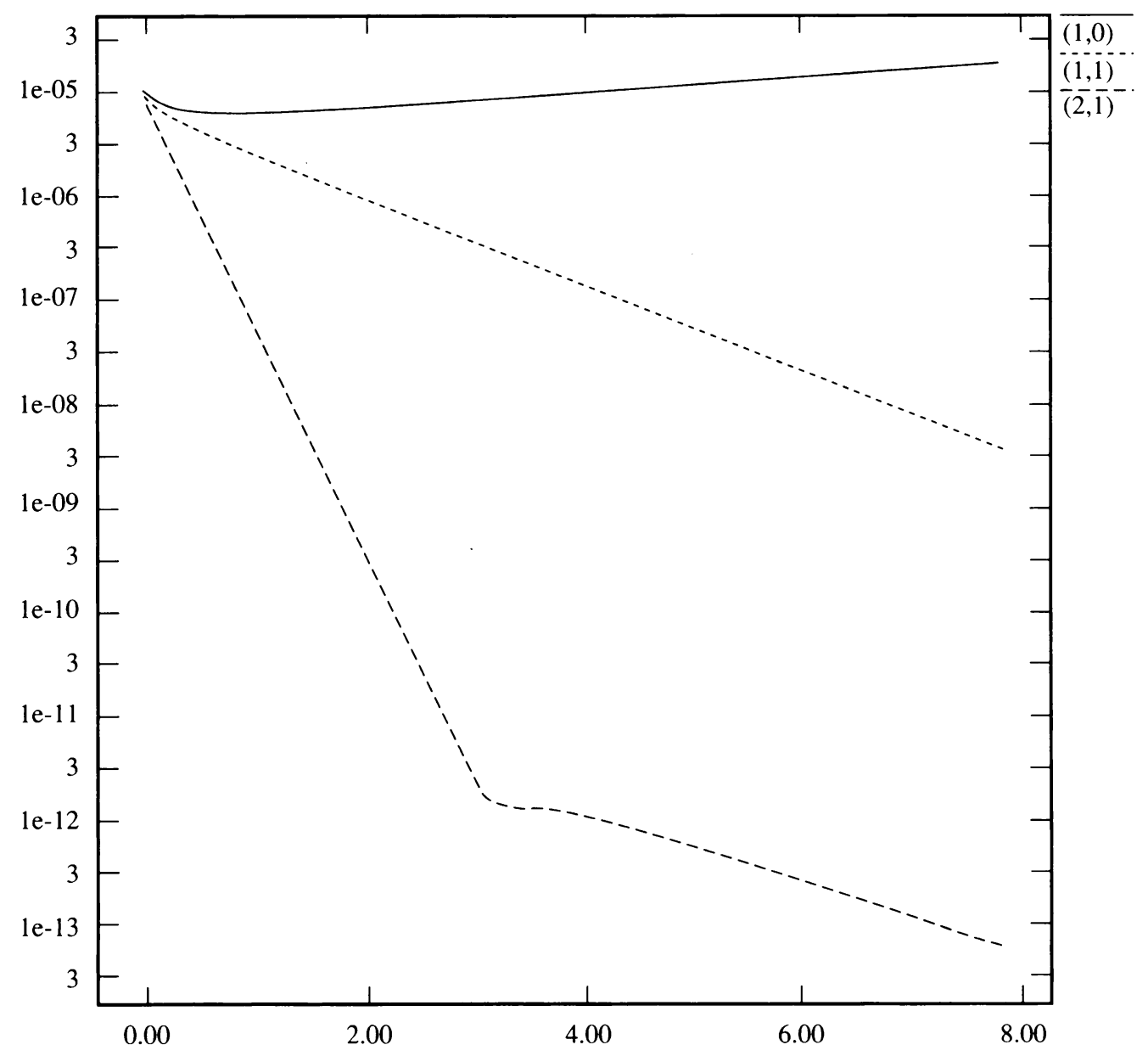

FIG. 4.5. Agreement of results obtained using our two-dimensional splitting scheme with the linear stability predictions for $q=0.9$. Here we plot the logarithm of $F(k, l, t)$ for the modes $(1,0)$, $(1,1)$, and $(2,1)$, where $F(k, l, t)=\left|f_{k l}(t)\right|$.

TABLE 4.5. Comparison of linear stability predictions for the exponential growth factor, $\lambda_{+}^{\text {lin }}$, with experimental values, $\lambda_{+}^{\exp }$.

\begin{tabular}{|c|rr|rr|}
\hline & \multicolumn{2}{|c|}{$(1,0)$ mode } & \multicolumn{2}{c|}{$(1,1)$ mode } \\
$q$ & $\lambda_{+}^{\operatorname{lin}}$ & $\lambda_{+}^{\text {exp }}$ & \multicolumn{1}{c|}{$\lambda_{+}^{\text {lin }}$} & \multicolumn{1}{c|}{$\lambda_{+}^{\text {exp }}$} \\
\hline 1.01 & $<0$ & $<0$ & $<0$ & $<0$ \\
0.99 & 0.0113 & 0.0113 & $<0$ & $<0$ \\
0.90 & 0.0940 & 0.0930 & $<0$ & $<0$ \\
0.85 & 0.1264 & 0.1263 & $<0$ & $<0$ \\
0.72 & 0.1728 & 0.1736 & $<0$ & $<0$ \\
0.70 & 0.1757 & 0.1755 & 0.0114 & 0.0113 \\
\hline
\end{tabular}


TABLE 4.6. Comparison of results of Coullet, Gil, and Lega (C) and those obtained with our second-order splitting scheme (S). Here we have rescaled $d_{\mathrm{m}}$ and $l_{c}$ to convert distances in Coullet's length 50 box to those in our $2 \pi$-periodic box.

\begin{tabular}{|c|c|c|c|}
\hline & $\langle N\rangle$ & $d_{\mathrm{m}}$ & $l_{\mathrm{c}}$ \\
\hline $\mathrm{C}$ & 34.1 & 0.55 & 0.70 \\
\hline $\mathrm{S}$ & 35.7 & 0.53 & 0.67 \\
\hline
\end{tabular}

The second test performed on our two-dimensional splitting scheme involved a comparison with results which Coullet, Gil, and Lega [5] obtained using a spectral method. They studied turbulence in the two-dimensional CGL equation for various parameter sets and presented results for the correlation length $l_{\mathrm{c}}$, the mean number of defects or spirals $\langle N\rangle$, and the mean nearest-neighbor distance between defects $d_{\mathrm{m}}$. In Table 4.6, we compare our results for these quantities, obtained using the two-dimensional splitting scheme, with those presented in [5], for the parameters $q=\sqrt{2 / 3} \cdot(2 \pi / 50), \rho=1 / 3, c_{0}=1 / 2(\mu=1, \alpha=2, \beta=-3$ in [5]). It can be seen that for this fairly energetic case of two-dimensional amplitude turbulence the agreement is good.

The performance of our two-dimensional implementation of the splitting method (3.32) on the tests just described indicates that, given sufficiently fine spatial and temporal discretizations, the scheme used will correctly solve the two-dimensional CGL equation for any parameter set of interest.

5. Efficiency of second-order method. We now show that our second-order splitting scheme for the one-dimensional CGL equation is computationally more efficient than standard spectral-type schemes, particularly when high spatial resolution is required.

For the parameters $q=0.14, \rho=c_{0}=0.25$, we compared the performance of our second-order splitting method with that of pseudospectral methods employing fourth-order Runge-Kutta and second-order predictor-corrector time-stepping schemes. We found that the predictor-corrector scheme was generally less efficient than our method, because it was slightly less accurate and required twice as many FFTs per time step. We also found that, despite its high accuracy, numerical stability requirements made the Runge-Kutta scheme much less efficient than our splitting scheme for $N \geq 256$. These results are shown in Table 5.1 (see p. 332). The error measure used was the r.m.s. difference at the gridpoints, i.e., the same as in the temporal convergence tests.

We note that the two main advantages of our method are its excellent stability properties and the fact that it gives second-order accuracy while requiring only two FFTs per time step, the equivalent of one evaluation of $\partial A / \partial t$. 
TABLE 5.1. Comparison of second-order splitting method (spl) with Runge-Kutta and predictor-corrector pseudospectral methods. The time steps $\Delta t$ were chosen to give numerical stability and an r.m.s. error of at most $O\left(10^{-10}\right)$ after ten time units when compared with the result for $\Delta t=10^{-4}$. The CPU times $t$ and r.m.s. errors $E$ are normalized by those of the splitting scheme.

\begin{tabular}{|r|c|ccc|ccc|}
\hline \multirow{2}{*}{$N$} & & \multicolumn{2}{|c|}{ Predictor-Corrector } & \multicolumn{3}{|c|}{ Runge-Kutta } \\
& $\Delta t_{\text {spl }}$ & $\Delta t$ & $t$ & $E$ & $\Delta t$ & $t$ & $E$ \\
\hline 64 & $1 \cdot e-3$ & $4 \cdot e-4$ & 8.18 & 1.8 & $5 \cdot e-2$ & 0.14 & $3.2 e-1$ \\
256 & $1 \cdot e-3$ & $4 \cdot e-4$ & 6.21 & 1.5 & $5 \cdot e-3$ & 1.03 & $3.3 e-5$ \\
1024 & $1 \cdot e-3$ & $2 \cdot e-4$ & 17.0 & 0.4 & $5 \cdot e-4$ & 12.6 & $7.5 e-7$ \\
\hline
\end{tabular}

Acknowledgments. The work reported here was supported by DARPA-URI contract N00014-86-K0754 and NSF grant IRI-9116451, and some of the computations were carried out at the Pittsburgh Supercomputer Center. We gratefully acknowledge helpful conversations with T. Kaper and R. Everson and the assistance of J. D. Rodriguez.

\section{REFERENCES}

[1] M. Bartuccelli, P. Constantin, C. R. Doering, J. D. Gibbon, and M. Gisselfalt, Hard turbulence in a finite dimensional dynamical system?, Phys. Lett. A 142, 349-356 (1989)

[2] M. Bartuccelli, P. Constantin, C. R. Doering, J. D. Gibbon, and M. Gisselfalt, On the possibility of soft and hard turbulence in the complex Ginzburg-Landau equation, Phys. D 44, 421-444 (1990)

[3] M. Bartuccelli, P. Constantin, C. R. Doering, J. D. Gibbon, and M. Gisselfalt, Errata, Phys. Lett. A 145, 476 (1990)

[4] A. J. Bernoff, Slowly varying fully nonlinear wavetrains in the Ginzburg-Landau equation, Phys. D 30, 363-381 (1988)

[5] P. Coullet, L. Gil, and J. Lega, A form of turbulence associated with defects, Phys. D 37, 91-103 (1989)

[6] E. Forest and R. D. Ruth, Fourth-order symplectic integration, Phys. D 43, 105-117 (1990)

[7] A. V. Gaponov-Grekhov and M. I. Rabinovich, Theory of dynamic turbulence (A. H. Luther, ed.), Advances in Theoretical Physics, Pergamon Press, New York, 1988, pp. 177-192

[8] R. H. Hardin and F. D. Tappert, Applications of the split-step Fourier method to the numerical solution of nonlinear and variable coefficient wave equations, SIAM Rev. 15, 423 (1973)

[9] A. Hasegawa and F. Tappert, Transmission of stationary nonlinear optical pulses in dispersive dielectric fibers. I. Anomalous dispersion, Appl. Phys. Lett. 23, 142-144 (1973)

[10] B. M. Herbst and F. Varadi, Symplectic methods for the nonlinear Schrödinger equation, preprint, 1992

[11] W. H. Louisell, M. Lax, G. P. Agrawal, and H. W. Gatzke, Simultaneous forward and backward integration for standing waves in a resonator, Appl. Optics 18, 2730-2731 (1979)

[12] R. I. McLachlan and P. Atela, The accuracy of symplectic integrators, Nonlinearity 5, 541-562 (1992)

[13] J. V. Moloney, M. R. Belic, and H. M. Gibbs, Calculation of transverse effects in optical bistability using fast Fourier transform techniques, Optics Comm. 41, 379-382 (1982)

[14] S.-C. Sheng and A. E. Siegman, Nonlinear-optical calculations using fast-transform methods: second-harmonic generation with depletion and diffraction, Phys. Rev. A 21, 599-606 (1980)

[15] L. Sirovich, J. D. Rodriguez, and B. Knight, Two boundary value problems for the Ginzburg-Landau equation, Phys. D 43, 63-76 (1990)

[16] T. R. Taha and M. J. Ablowitz, Analytical and numerical aspects of certain nonlinear evolution equations. II. Numerical, nonlinear Schrödinger equation, J. Comput. Phys. 55, 203-230 (1984) 
[17] F. Tappert, Numerical solutions of the Korteweg-de Vries equation and its generalizations by the split-step Fourier method, Nonlinear Wave Motion (A. C. Newell, ed.), Amer. Math. Soc., Providence, RI, 1974, pp. 215-216

[18] F. D. Tappert and C. N. Judice, Recurrence of nonlinear ion acoustic waves, Phys. Rev. Lett. 29, 1308-1311 (1972)

[19] J. A. C. Weideman and B. M. Herbst, Split-step methods for the solution of the nonlinear Schrödinger equation, SIAM J. Numer. Anal. 23, 485-507 (1986)

[20] H. Yoshida, Construction of higher order symplectic integrators, Phys. Lett. A 150, 262-268 (1990) 